\title{
Detectable viral load among HIV -1 positive pregnant women on ART (Option B +) in northern Tanzania: Baseline results from the HIVDR study.
}

\author{
Nicholaus Steven Mazuguni a, b, Festo Mazuguni b, Eva Prosper Muro a, b, c \\ Kilimanjaro Christian Medical Centre (KCMC), Moshi, Tanzania \\ Kilimanjaro Christian Medical University College (KCMU-Co), Moshi, Tanzania \\ Kilimanjaro Clinical Research Institute (KCRI), Moshi, Tanzania \\ *Correspondence to Dr. Eva Prosper Muro (e.muro@kcri.ac.tz)
}

\begin{abstract}
Introduction: In Tanzania, the Ministry of Health, Community Development, Gender, Elderly and Children (MoHCDEC) has implemented the Option B+ as one of the strategies to facilitate achievement of elimination of mother to child transmission of HIV. To prevent emergence of drug resistance mutations early identification of option B+ failure is critical. The emergence of drug resistance mutation and subsequent treatment failure poses a major concern for HIV program in low and middle-income resource settings where treatment options are limited.

Methodology: We recruited treatment naive, treatment experienced HIV-1 positive pregnant women and those who had prophylaxis in their previous pregnancy in Kilimanjaro, northern Tanzania August 2016 to February 2017. Whole blood (2ml) for biochemistry, viral load and drug resistance testing were taken at baseline. ARV drug resistance testing was done on women with $\mathrm{VL} \geq 1000$ copies $/ \mathrm{ml}$. We used descriptive statistic and logistic regression to determine the strength of association between virologic outcome (virologic failure) and independent predictors.

Results: One hundred and forty eight (148) pregnant HIV-positive women were enrolled in the study with mean age of 29.82 years (SD=6.17) from August, 2016 to February, 2017. Virologic failure was demonstrated in 34 (23\%) with viral load $\geq$ 1,000 copies $/ \mathrm{ml}$. Genotyping results were available from 26 women, mutations associated with ARV resistance were detected in $23.1 \%(n=6 / 26)$. Among the six women with ARV resistance mutation $4(66.7 \%)$ had high level resistance and $2(33.3 \%)$ had low level resistance. Among the 26 samples genotyped 15(58\%) viruses were subtype A, while eight were subtype C $(31 \%)$ and three subtypes $D(11 \%)$. The most dominant drug resistance mutations against the reverse transcriptase inhibitors for the women with high level resistance were K103N, Y188L, D67N, K70R, M184V, T215F, K219EQ, and the low-level resistance was E138A. The older age was associated with virological failure compared to those who were $<20$ year of age.

Conclusion: Viral load testing should be done on women who were already on antiretroviral treatment on their first antenatal visit to ensure early detection of virological failure and enable clinicians to take an appropriate course of action on their management.

Educational intervention on adherence should be targeted at an early stage to women with virological failure during pregnancy to reduce the emergence of HIV-1 drug resistance mutations.
\end{abstract}

Keywords: PMTCT, ART, HIV, pregnant women, viral load suppression, HIV drug resistance, Tanzania

\section{INTRODUCTION}

Cince 2012, the World Health Organization (WHO) Srecommends using lifelong antiretroviral therapy

(ART) for all pregnant and breastfeeding women regardless of $\mathrm{CD} 4$ counts and clinical stage, and provision of nevirapine or zidovudine to all HIV- exposed infants for four to six weeks regardless of the feeding method. These recommendations are known as "Option B+". In 2013, the WHO consolidated antiretroviral (ARV) guidelines that endorsed this approach, particularly in HIV-endemic resourcelimited settings. ${ }^{2}$ These guidelines also promoted the 
use of viral load (VL) testing as the preferred approach to monitoring ART and identifying treatment failure, recommending testing six months after starting ART and every 12 months thereafter.

In September 2013, the Tanzanian government initiated a policy of lifelong ART for all HIV-infected pregnant women for prevention of mother-to-child transmission (PMTCT) regardless of CD4 count or clinical status (also known as Option $\mathrm{B}+$ ). ${ }^{3}$ In the Kilimanjaro region, Northern Tanzania, Option B+ was deployed in April 2014. There are limited VL data available from programs implementing "Option B+," lifelong ART to all HIV-positive pregnant and postpartum women, in resource-limited settings. The emergence of drug resistance and subsequent treatment failure poses a major concern for HIV programs in low resource settings where treatment options are limited. The most challenging issue, particularly with Option $\mathrm{B}+$, is combined antiretroviral therapy (cART) adherence because these women are relatively healthy and therefore less motivated to accept cART inconveniencies.

Suboptimal adherence may promote HIV drugresistance (HIVDR) development. ${ }^{4}$ Furthermore, the implementation of Option $\mathrm{B}+$ would have major programmatic implications: scale-up of women receiving ART from pre-conception will rapidly increase with 2015 WHO recommendations for ART initiation in all HIV- positive pregnant and nonpregnant women regardless of their CD4 cell count. ${ }^{5}$ However, empirical data to adequately inform policy are currently lacking, in particular as to what extent $\mathrm{B} / \mathrm{B}+$ could result in increased rates of $\mathrm{ARV}$ drug resistance. In this study, the overall objective was to assess the virological failure and drug resistance mutations in ART naïve and ART experienced HIV-1 pregnant women.

\section{MATERIALS AND METHODS}

\section{Study Design}

The HIVDR study was a descriptive cross-sectional study of 148 HIV-positive pregnant eligible women. We enrolled women falling in any of the following three categories: treatment naïve pregnant women who have initiated ART for the first time, secondly those who were previously enrolled through PMTCT program either through prophylaxis or treatment (option A\& B) and lastly were pre-ART HIV-linfected pregnant women who had been initiated cART through care and treatment services before the current pregnancy.

\section{Study Population}

Between August 2016 and February 2017, eligible HIV- positive pregnant women were enrolled from three study sites, Kilimanjaro Christian Medical Centre (KCMC) and Pasua, and Himo Health centres. Eligibility criteria included documented HIV infection, women aged $\geq 18$ years old, HIV-1 positive pregnant women with a first HIV-l positive test during the current pregnancy, HIV- 1 positive pregnant women with previous history of ART exposure for PMTCT or for post-exposure prophylaxis (PEP), those who have been on cART through care and treatment centre (CTC) program and willing to provide informed consent for herself.

\section{Data Collection}

Women underwent an enrolment interview, which included demographic information, HIV and ARTrelated history, use of alcohol and concomitant drug use. Medical history and liver function tests were done. Responses were documented on a case report form (CRF). Whole blood $(2 \mathrm{ml})$ for biochemistry, VL and HIVDR testing were taken at baseline. Data were reviewed for completeness and quality, and entered into the study database. RNA-PCR testing for VL in women was done at the time of enrolment by the clinical laboratory at KCMC using COBAS AmpliPrep/COBAS TaqMan (manufacturer Roche Platform) HIV-1 Test, version 2.0 (v2.0) with a lower detection limit at 20 copies $/ \mathrm{ml}$. ARV drug resistance testing was conducted on women with $\mathrm{VL} \geq 1000$ copies/ml at National Health Laboratory. Quality Assurance and Training Centre in Dar es Salaam for HIVDR genotyping testing.

Informed consent was obtained and documented via signature and all eligible women were enrolled in the study. All study staff received training on data collection and on all ethical issues regarding research with human subjects. Ethical approvals were obtained from the KCMC College Research Ethics Committee, in Moshi, Tanzania and by the National Health Research Ethics Committee at the National Institute for Medical Research in Dar-es Salaam, Tanzania.

\section{Statistical analysis}

Descriptive statistics were used to summarize demographics. Logistic regression analysis was performed and an odds ratio with 95\% confidence interval $(\mathrm{CI})$ was used to determine the strength of association between virologic outcome (virologic failure) and independent predictors. All statistical tests were two sided and the level of statistical significance 
was set at $\mathrm{P}<0.05$ (2-tailed). The crude analysis assessed the association between individual characteristics of the women and the outcome variable (HIV-1 VL). Then data was analysed using the statistical package for social science (SPSS version 20) IBM Cooperation by Armonk, New York, United States of America.

\section{RESULTS}

\section{Demographic Characteristics}

A total of 148 pregnant HIV-positive women were enrolled in the study with a mean age of 29.82 years $(S D=6.17)$ from August 2016 to February 2017. The newly diagnosed HIV pregnant women were $82(55.4 \%)$ and previously diagnosed from CTC were $39(26.4 \%)$ and $27(18.2 \%)$ were those given prophylaxis in their previous pregnancy (Table 1 ). The median ART duration for women who were already enrolled in CTC was 4.5 years with a range of 3 months to 11 years and 6 months among those women who were previously diagnosed in CTC. Most (2-5\%) HIV positive pregnant women with active TB were distributed across all the treatment arms.

\section{Viral load}

Detectable VL was >1000 copies/ml among newly diagnosed HIV - 1 infected pregnant women compared to those who were previously on ART before the current pregnancy and those who had prophylaxis in their previous pregnancy $(<1000$ copies/ml) and this was found to be statistically significant $(\mathrm{P}=0.006)$ (Table 1). Virologic failure was demonstrated in $23 \%$ overall (Table 2).

TABLE 1: Characteristics of HIV positive pregnant women-initiated ART under option B+ by virologic outcome at study enrolment ( $\mathrm{N}=148)$

\begin{tabular}{|c|c|c|c|}
\hline \multirow[b]{2}{*}{ Variables } & \multicolumn{2}{|c|}{ HIV viral load (copies/mls ) } & \multirow[b]{2}{*}{$\begin{array}{c}\chi 2 \\
\text { P-value }\end{array}$} \\
\hline & $\begin{array}{c}<1000 \text { copies } / \mathrm{ml} \\
(\mathrm{n}=114)\end{array}$ & $\begin{array}{c}>1000 \text { copies } / \mathrm{ml} \\
(\mathrm{n}=34)\end{array}$ & \\
\hline \multicolumn{4}{|l|}{ Hospital site } \\
\hline Kilimanjaro Christian Medical Centre & $53(77.9)$ & $15(22.1)$ & \multirow{3}{*}{.359} \\
\hline Himo Health Centre & $27(84.4)$ & $5(15.6)$ & \\
\hline Pasua Health Centre & $34(70.8)$ & $14(29.2)$ & \\
\hline \multicolumn{4}{|l|}{ Age (Years) } \\
\hline$<20$ & $4(80.0)$ & $1(20.0)$ & \multirow{4}{*}{.744} \\
\hline $20-24$ & $22(84.6)$ & $4(15.4)$ & \\
\hline $25-29$ & $30(73.2)$ & $11(26.8)$ & \\
\hline 30 and above & $58(76.3)$ & $18(23.7)$ & \\
\hline \multicolumn{4}{|l|}{ Gestational age at screening } \\
\hline 1st trimester (6-12 weeks) & $17(81)$ & $4(19)$ & \multirow{3}{*}{.179} \\
\hline 2nd trimester (13-24 weeks) & $50(70.4)$ & $21(29.6)$ & \\
\hline 3rd trimester (25-40 weeks) & $47(83.9)$ & $9(16.1)$ & \\
\hline \multicolumn{4}{|l|}{ Index pregnancy and ART initiation* } \\
\hline During pregnancy & $79(72.5)$ & $30(27.5)$ & \multirow[t]{2}{*}{.02} \\
\hline Before pregnancy & $35(89.7)$ & $4(10.3)$ & \\
\hline \multicolumn{4}{|l|}{ Anti TB* } \\
\hline Yes & $5(100)$ & - & \multirow{2}{*}{.266} \\
\hline No & $109(76.2)$ & $34(23.8)$ & \\
\hline \multicolumn{4}{|l|}{ Alcohol* } \\
\hline Yes & $25(80.6)$ & $6(19.4)$ & \multirow{2}{*}{.810} \\
\hline No & $89(76.1)$ & $28(23.9)$ & \\
\hline \multicolumn{4}{|l|}{ Treatment arms } \\
\hline Newly diagnosed & $55(67.1)$ & $27(32.9)$ & \multirow{3}{*}{.006} \\
\hline CTC & $35(92.1)$ & $4(7.9)$ & \\
\hline PMTCT & $24(85.7)$ & $3(14.3)$ & \\
\hline
\end{tabular}

*Fischer exact test 
TABLE 2: Factors associated with virologic outcome among HIV pregnant women-initiated ART under option $\mathrm{B}+(\mathrm{N}=148)$

\begin{tabular}{|c|c|c|c|c|}
\hline \multirow[b]{2}{*}{ Variable } & \multirow[b]{2}{*}{ Total } & \multirow[b]{2}{*}{$\begin{array}{c}\text { Viral load } \geq \\
\text { 1000copies } / \mathrm{ml} \text { n }(\%)\end{array}$} & \multicolumn{2}{|c|}{ Crude analysis } \\
\hline & & & $\begin{array}{c}\text { Odds ratio (OR) } \\
(95 \% \mathrm{CI})\end{array}$ & P-value \\
\hline Total & 148 & $34(23)$ & & \\
\hline \multicolumn{5}{|l|}{ Age group } \\
\hline$<20$ & 5 & $1(20.0)$ & Ref & \\
\hline $20-24$ & 26 & $4(15.4)$ & $0.73(0.06-8.32)$ & .85 \\
\hline $25-29$ & 41 & $11(26.8)$ & $1.47(0.15-14.59)$ & .744 \\
\hline 30 and above & 76 & $18(23.68)$ & $1.24(0.13-11.83)$ & .798 \\
\hline \multicolumn{5}{|l|}{ Gestational age } \\
\hline $\begin{array}{l}\text { 1st trimester (6-12 } \\
\text { weeks) }\end{array}$ & 21 & $4(19.05)$ & Ref & \\
\hline $\begin{array}{l}2^{\text {nd }} \text { trimester }(13-24 \\
\text { weeks) }\end{array}$ & 71 & $21(29.58)$ & $1.79(0.54-5.94)$ & \\
\hline $\begin{array}{l}3^{\text {rd }} \text { trimester }(25-40 \\
\text { weeks })\end{array}$ & 56 & $9(19.07)$ & $0.81(0.22-2.99)$ & .756 \\
\hline \multicolumn{5}{|l|}{ Alcohol } \\
\hline No & 117 & $28(23.93)$ & Ref & \\
\hline Yes & 31 & $6(19.35)$ & $0.76(0.28-2.05)$ & .591 \\
\hline \multicolumn{5}{|l|}{ Exposed ART } \\
\hline Yes & 25 & $5(20)$ & Ref & \\
\hline No & 123 & $29(23.58)$ & $1.23(0.43-3.58)$ & .699 \\
\hline \multicolumn{5}{|l|}{ ALT (normal) } \\
\hline Yes & 144 & $32(22.2)$ & Ref & \\
\hline No & 2 & $1(50)$ & $3.5(0.21-57.53)$ & .380 \\
\hline \multicolumn{5}{|l|}{ AST (normal) } \\
\hline Yes & 120 & $27(22.5)$ & Ref & \\
\hline No & 26 & $6(23.08)$ & $1.03(0.38-2.23)$ & .949 \\
\hline
\end{tabular}

\section{Antiretroviral Drug Resistance}

There were 34 (23\%) with VL $\geq 1,000$ copies/ml for genotyping for ARV drug resistance mutations. The VL among these women with genotyping results was 1038 to 242208 copies/ml. Genotyping results were available from 26 women, mutations associated with ARV resistance were detected in 6(23.1\%). Among the six women with ARV resistance mutation $4(66.7 \%)$ had high level resistance and 2(33.3\%) had low level resistance. Three women were previously diagnosed in the CTC, one woman was previously exposed to ART during her previous pregnancy and two were newly diagnosed. Among the 26 samples genotyped $15(58 \%)$ viruses were subtype A, while eight were subtype C $(31 \%)$ and three subtype D $(11 \%)$. The most dominant drug resistance mutations against the reverse transcriptase inhibitors for the women with high level resistance were K103N, Y188L, D67N, K70R, M184V, T215F, K219EQ (Figure 1) and for the low-level resistance it was E138A.

\section{Determinants of virological failure}

In the crude analysis the following significant predictors for VL $\geq 1000$ copies/ml were identified: Age at ART initiation, gestational age at ART initiation, previous exposure to ART, alcohol use, use of anti TB medications and liver function test AST and ALT (Wald test, $\mathrm{P}$-value $<0.05$ ). At bivariate analysis none of these variables were associated with VL $\geq 1000$ copies $/ \mathrm{ml}$. However, increase in age above 20 years was associated with the odds of viral load $\geq$ 1000 copies $/ \mathrm{ml}$ (ORcrude $=0.73,95 \% \mathrm{CI}=0.06$ to 8.32 ) and (ORcrude $=1.47,95 \% \quad \mathrm{CI}=0.15$ to 14.59 ) compared to those who were less than 20 years of age but the differences were not statistically significant (Table 2).

\section{Virologic failure sub-group analysis}

In a sub-group analysis of the factors associated with virologic failure among previously diagnosed HIV pregnant women in CTC, $7.7 \%$ (3/39) had VL $\geq$ 
1000copies $/ \mathrm{ml}$. All these women had dual high level of resistance mutations to the nucleoside reverse transcriptase inhibitors (NRTIs) and the nonnucleoside reverse transcriptase inhibitors

(NNRTIs). Intermediate drug resistance mutations were also present in two among the three women, one had etravirine and the other one had tenofovir drug resistance mutations. All these HIV positive pregnant women had high levels of resistance mutations to the NRTI's lamivudine and Emtricitabine. One woman had high levels of resistance mutations to another NRTIs Zidovudine. All the three women were found to have high levels of resistance mutations to NNRTIs Nevirapine and Rilpivirine, two women on Efavirenz and one woman on Etravirine. All these women had subtype A viral infection with both NNRTIs and NRTIs HIVDR mutations (Figure 2).
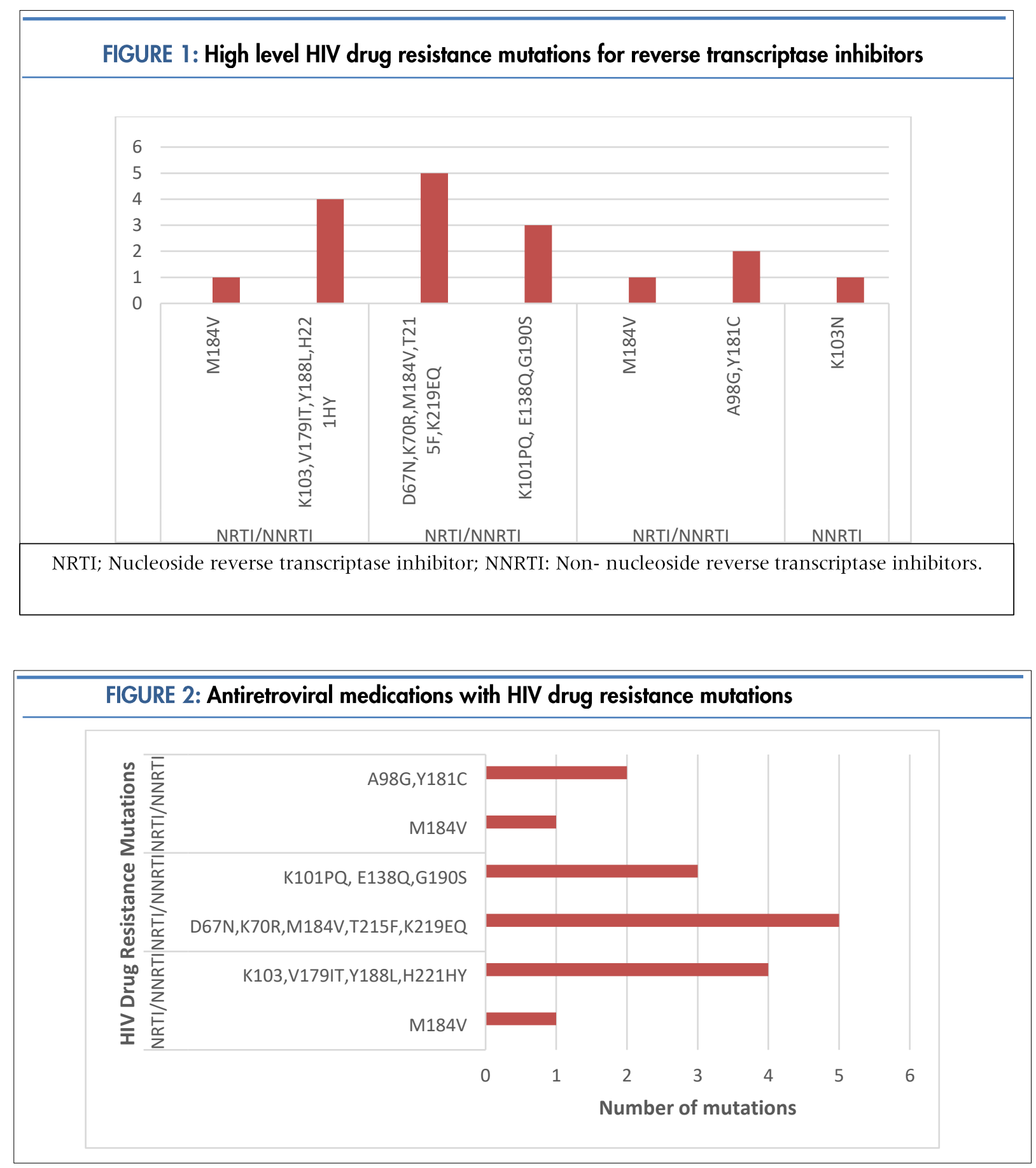


\section{DISCUSSIONS}

In this descriptive cross-sectional study conducted to estimate the proportion of HIV pregnant women with $\mathrm{VL} \geq 1000$ copies/ml and to identify the clinical factors associated with virologic outcome, we found an overall proportion of $\mathrm{VL} \geq 1000$ copies $/ \mathrm{ml}$ of $23 \%$. Amongst the three treatment arms the newly diagnosed HIV pregnant women had the highest proportion of $\mathrm{VL}$ $\geq 1000$ copies $/ \mathrm{ml}(\mathrm{P}=0.006)$ compared to women who were previously initiated on ART and those who had taken prophylaxis for PMTCT in their previous pregnancy. We found six women with HIVDR mutation in the current study. Among the six women three of them who were previously on ART had virological failure and this poses a concern in our setting because in routine ANC pregnant women already receiving ART are continued on ART without VL testing. HIV- infected pregnant women with high VL are at risk of transmitting HIV and drug -resistant mutations to their infants. ${ }^{6}$ Our findings showed that $8 \%$ of women had a VL $\geq 1000$ copies $/ \mathrm{ml}$ and were receiving $\mathrm{ART}$ at the time of their first ANC . A recent study done in Malawi showed $10 \%$ of women receiving $\mathrm{ART}$ for $\geq 6$ months presenting to ANC were not virally suppressed. ${ }^{7}$ The number of pregnant women receiving universal ART reporting for ANC for the first time is likely to increase. Targeted antenatal HIV RNA testing provides an opportunity to identify virological failure and minimize the risk of mother - to - child transmission.

The low percentage of women with treatment failure at the ANC suggests that Tanzania has made progress towards achieving the last $90 \%$ of the UNAIDS 90-9090 targets among women for control of the HIV epidemic. ${ }^{8}$ Although not statistically significant, we also observed a trend towards higher rates of viral load suppression in the younger age group ( $\leq 20$ years). HIVDR mutations have been reported in Tanzania, some with thymidine analogue associated mutations which compromise susceptibility to NRTIs. Early ART failure and drug resistance were observed among two newly diagnosed treatment naïve HIV pregnant women. Switching to second-line ART without programmatic HIVDR drug resistance testing, which is a common practice, may transfer cross-resistance patterns to newly switched drugs and limit available treatment options. Initiating treatment early and ensuring optimal adherence are vital for the success and durability of first-line ART in these settings. The most common HIV drug resistance DR mutations in this study were against the reverse transcriptase inhibitors (K103N, Y188L, D67N, K70R, M184V, T215F, K219EQ and E138A) which are similar to those reported in resistance studies from other SSA and Tanzanian settings. 9-12

All the observed mutations are associated with the ARV drugs with a low genetic barrier to resistance (lamivudine, nevirapine and efavirenz) that are commonly used in SSA and Tanzania.

An important finding in this study, although limited by the small numbers, was pre HIVDR mutation in two women at baseline. Several studies have shown an association between pre-therapy resistance and an increased risk of ART failure. 13-16 This study has some limitations. Although the findings highlight several successful aspects of the program, all study facilities are high-volume where CTCs are integrated in the ANC sites located in the Kilimanjaro region. The study enrolled only women in the three sites in Kilimanjaro. However, our study fulfils a critical need for evaluation of VL suppression data among ARTexperienced HIV positive pregnant women and ARTnaïve women in ANC in an established Option B+ program. Future studies on HIV VL and drug resistance mutation testing for those with high VL should be planned as an optimal approach to limit transfer of cross-resistance patterns to newly switched drugs and limit available treatment options.

\section{CONCLUSIONS}

VL testing should be done on women who were already on ART on their first antenatal visit to ensure early detection of virological failure. This will enable clinicians to take an appropriate course of action on their management. Educational intervention on adherence among HIV-1 positive pregnant women on ART should be targeted at an early stage to those with virological failure during pregnancy to reduce the emergence of HIVDR mutations.

Acknowledgement: The authors thank the Medical Education Partnership Initiative (MEPI) Tanzania for funding the project through MEPI-Junior faculties' fellowship. We would like to thank the KCRI Biotechnology laboratory for the biochemistry analysis and processing of the samples for phylogenetic analysis. We would also like to thank the Clinical laboratory at KCMC for the

viral load testing. The United Republic of Tanzania, MOHCDGEC, the National Health Laboratory Quality Assurance and Training Centre for HIV -1 drug resistance genotyping testing. Sincere thanks to the research team at KCMC, Pasua and Himo Health Centre, and above all to the participants in the study. 


\section{Financial support}

The study was funded by the Fogarty International Centre of the National Institutes of Health under Award Number D43Tw010138. The funders had no role in study design, data collection and analysis, decision to publish, or preparation of the manuscript.

\section{Conflict of interest}

There was no conflict of interest

\section{REFERENCE}

1. World Health Organization. (2012). Programmatic update: use of antiretroviral drugs for treating pregnant women and preventing HIV infection in infants: executive summary. World Health Organization. https:/lapps who int/iris/handle/10665/70892 2012.

2. Consolidated guidelines on the use of antiretroviral drugs for treating and preventing HIV infection: recommendations for a public health approach.World Health Organization, Geneva, Switzerland. 2013.

3. Tanzania National Guidelines for Comprehensive Care Services for PMTCT and keeping Mothers Alive (Option B+). 1-172. Tanzania Ministry of Health and Social Welfare 2013.

4. Mepham S, Zondi Z, Mbuyazi A, Mkhwanazi N, Newell ML. Challenges in PMTCT antiretroviral adherence in northern KwaZulu-Natal, South Africa. AIDS Care 2011,23:741-747 2011.

5. World Health Organization. Guideline on when to start antiretroviral therapy and on pre-exposure prophylaxis for HIV. Geneva, Switzerland: WHO Department of HIV/AIDS. WHO Department of HIV/AIDS; 2015.

6. Zeh C, Weidle PJ, Nafisa L, et al. HIV-1 drug resistance emergence among breastfeeding infants born to HIV-infected mothers during a single-arm trial of triple-antiretroviral prophylaxis for prevention of mother-to-child transmission: a secondary analysis. PLoS medicine. 2011;8(3):e1000430. doi: 10.1371 /journal. pmeid:1000430

7. Chagomerana MB, MillerWC, TangJ H, et al. (2018)Prevalence of antiretroviral therapy treatment failure amongHIV-infected pregnant women at first antenatal care: PMTCT Option B+ in Malawi. PLoSONE. 2018; 13(12):e0209052.

8. UNAIDS(2017)Ending AIDS: Progress toward the 90-90-90. 2017.

9. Hamers RL, Sigaloff KC, Wensing AM et al.; Patterns of HIV-1 drug resistance after first-line antiretroviral therapy (ART) failure in 6 sub-Saharan African countries: implications for second-line ART strategies. Clin Infect Dis. 2012; 54: 16601669. doi:10.1093/cid/cis254.

10. Fofana DB, Soulié C, Baldé A, et al., High level of HIV-1 resistance in patients failing long-term first-line antiretroviral therapy in Mali. J Antimicrob Chemother. 2014;69(9):25312535. doi:10.1093/jac/dku153

11. Ramadhani HO, Thielman NM, Landman KZ et al., Predictors of incomplete adherence, virologic failure, and antiviral drug resistance among HIV-infected adults receiving antiretroviral therapy in Tanzania. Clin Infect Dis. 2007; 45(11): 1492-1498. d.oi:10.1086/522991.

12. Ngarina M, Kilewo C, Karlsson $\mathrm{K}$, et al., Virologic and immunologic failure, drug resistance and mortality during the first 24 months postpartum among HIV-infected women initiated

on antiretroviral therapy for life in the Mitra plus Study, Dar es Salaam, Tanzania. BMC Infect Dis 2015; 15: 175. doi:10.1186/s12879-015-0914-Z.
13. Hamers RL, Schuurman R, Sigaloff KC, et al., Effect of pretreatment HIV-1 drug resistance on immunological, virological, and drug-resistance outcomes of first-line antiretroviral treatment in sub-Saharan Africa: a multicentre cohort study. Lancet Infect Dis. 2012; 12:307-317. doi:10.1016/S1473-3099(11)70255-9.

14. Wittkop L, Günthard HF, de Wolf F, et al., Effect of transmitted drug resistance on virological and immunological response to initial combination antiretroviral therapy for HIV (EurocoordCHAIN joint project): a European multicohort study. Lancet Infect Dis. 2011; 11(5): 363-371. 2011doi:10.1016/S1473 3099(11)70032-9

15. Li JZ, Paredes R, Ribaudo HJ, et al., Relationship between minority nonnucleoside reverse transcriptase inhibitor resistance mutations, adherence, and the risk of virologic failure. AIDS. 2012; 26(2): 185-192. doi: 10.1097/QAD.0b013e3283e9d7d.

16. Hawkins C, Ulenga N, Liu E, et al., HIV virological failure and drug resistance in a cohort of Tanzanian HIVinfected adults. J Antimicrob Chemother. 2016;71(7): 19661974. doi:10 1093/jac/dkw051.

Peer Reviewed

Competing Interests: None declared.

Received: 10/3/2020; Accepted: 3/10/2021

Cite this article as: Mazuguni NS, Mazuguni F, Muro EP. Virological failure among HIV-1 pregnant women. E Afr Sci. 2021;3(1):44-50. http://doi.org/10.24248/EASci-D-20-00009

(C) Mazuguni NS. This is an open-access a rticle distributed under the terms of the Creative Commons Attribution License, which permits unrestricted use, distribution, and reproduction in any medium, provided the original author and source are properly cited. To view a copy of the license, visit http://creativecommons.org/licens- es/by/4.0/. When linking to this article, please use the following permanent link: http://doi.org/10.24248/EASci-D-20-00009 